\title{
La inscripción de la angustia en el campo psicopatológi- co: referencias etimológicas, consideraciones filosóficas y categorías precursoras.
}

The inscription of the anguish in the psychopathological field: etymological references, philosophic considerations and precursors categories.

\section{Francisco Pizarro Obaida.}

${ }^{a}$ Psicólogo. Escuela de Psicología. Facultad de Ciencias Sociales. Universidad Andrés Bello (Chile).

Correspondencia: Francisco Pizarro Obaid (fpizarro@unab.cl)

Recibido: 27/05/2010; aceptado: 12/12/2010

RESUMEN: A diferencia de algunas categorías fundamentales que recorren la historia de la psicopatología, la incorporación de la angustia al marco nosográfico de la psiquiatría se producirá, pese a su relevancia clínica, de manera tardía, iniciándose su redefinición durante la segunda mitad del siglo XIX.

El presente trabajo identificará problemáticas relativas al campo semántico y etimológico de la angustia, para luego examinar las tesis sostenidas por Kierkegaard sobre el objeto y la temporalidad involucrados en dicha experiencia. Se postulará que estas referencias filosóficas serán claves en la redefinición y la inscripción del problema en el campo psicopatológico, pudiendo constatar su influencia en las categorías de delirio emotivo, agorafobia, miedo a los espacios y en la distinción entre miedo $\mathrm{y}$ angustia, formulada por Karl Jaspers.

PALABRAS CLAVE: angustia, ansiedad, Kierkegaard, psicopatología.
ABSTRACT: In difference to some fundamental categories that run over the history of psychopathology, the incorporation of the anguish to the nosographic frame of the psychiatry will occur in a tardy manner, in spite of its clinic relevance, starting its redefinition, during the second half of the XIX century.

The present work will identify problems related to the semantic and etymological field of the anguish, to examine later on, the theses supported by Kierkegaard regarding the object and the temporality involved in this experience. It will be postulated that these philosophical references will be the keys to the redefinition and the inscription of the problem in the psychopathological field, being able to prove its influence in the emotional delirium, agoraphobia and fear to the spaces categories and the distinction between fear and anguish, as formulated by Karl Jaspers.

KEY WORDS: anguish, anxiety, Kierkegaard, psychopathology.

\section{Introducción}

La tristeza, la culpa y el miedo han tenido una larga historia en los dominios de la filosofía, la medicina y la psicopatología general, siendo sistemáticos los esfuerzos por delimitar y especificar su naturaleza (1). Reunidos bajo la categoría de melancolía, es posible verificar que desde el siglo $\mathrm{V}$ a. c, dicha entidad participó del discurso médico y deambuló por las más diversas nosografías y marcos explicativos, transitando desde el saber hipocrático hasta los nuevos referentes clasificatorios $(2,3)$, donde, si bien se impone el término depresión, podemos evidenciar su huella. En contraste con dicha evolución, la aparición de la angustia, como pro- 
blema médico y más tarde psicopatológico, puede ser considerada como un hecho reciente.

Esto no implica que la angustia estuviese ausente de los avatares de la existencia humana. El análisis etimológico y semántico pondrá en evidencia la diversidad de sentidos y significados que le fueron asociados a través de los siglos, oscilando entre la primacía del registro corporal, bajo el signo del ahogo y la opresión, hasta la dimensión subjetiva donde confluirían el miedo, el terror y el horror. En una u otra dirección la angustia invoca sistemáticamente diversas formas de sufrimiento. ¿De qué modo esa experiencia que acompaña a lo humano desde los albores de la existencia se instituyó como un concepto de orden psicopatológico?

Durante la segunda mitad del siglo XIX surgieron una serie de indagaciones en torno a las problemáticas referidas al miedo y la angustia. La novedad clínica que se registra hacia 1890, es que síntomas tales como la opresión, la sudoración, el vértigo, las palpitaciones, los temblores, - que hasta entonces se asociaban a problemáticas médicas relativas al corazón, el oído, la visión o el estómago -, serán agrupados en una categoría clínica particular y autónoma perteneciente al campo psicopatológico (4).

Los trabajos pioneros que se desarrollaron principalmente en el ámbito de la psiquiatría francesa y alemana, darán cuenta de esta innovación al proponer categorías diagnósticas inéditas, tales como, el delirio emotivo [neurosis del sistema nervioso ganglionar visceral] de Morel (5); el temor de las plazas [agorafobia] de Westphal (6); la neuropatía cerebro-cardiaca de Krishaber (7); el miedo de los espacios de Legran du Saulle (8). Tras este primer impulso, desde Norteamérica, Da Costa (9) proponía la categoría de corazón irritable y, más tarde, G. Beard $(10,11)$, acuñaba el término neurastenia.

La ligazón entre nerviosidad, modernidad y angustia, promovida por Beard a partir de 1880 a través de la categoría de neurastenia, dará lugar a una discusión en el ámbito europeo (12), impulsando nuevos estudios, entre los que destacarán la conceptualización de Kaan (13) sobre las relaciones que guardan el afecto angustioso y la neurosis obsesiva; la proposición freudiana de la neurosis de angustia $(14,15)$; la identificación de estados angustiosos enmascarados en la neurastenia, por parte de Hecker $(16,17)$.

Definitivamente, la clínica de las enfermedades nerviosas le dará un lugar a la angustia en el territorio clasificatorio.

¿Cómo se habría producido la construcción de un concepto singular, diferenciándolo de ese universo del miedo, el susto, el pánico, el terror, el estremecimiento, del cual formó parte durante siglos? Propondremos que las nuevas distinciones llevadas a cabo en el campo psicopatológico de la segunda mitad del siglo XIX tuvieron como antecedente, implícito o explícito, a la reflexión filosófica sobre la angustia emprendida en 1884 por Kierkegaard (18). La oposición entre el miedo 
ORIGINALES Y REVISIONES

y la angustia, así como el análisis de la temporalidad involucrada en el fenómeno angustioso, servirán de referentes, no sólo a las posteriores discusiones filosóficas, sino que se instituirán como elementos precursores del desarrollo del campo de los trastornos referidos a la angustia. Como advertirá López- Ibor "la clínica de la angustia no puede hacerse sin su psicopatología y ésta es imposible realizarla sin las necesarias referencias filosóficas" (19, p. 99).

\section{Referentes etimológicos y semánticos}

Los términos angustia y ansiedad son las expresiones más recurrentes de la literatura psicopatológica $(2,3)$ para referirse a los fenómenos que reúnen signos neurofisiológico relativo a la opresión, el ahogo y el vértigo e incluyen un correlato subjetivo referido a la posibilidad de la muerte, la locura o la enfermedad. Frecuentemente son términos utilizados de modo indistinto $\mathrm{y}$, a menudo, se los considera equivalentes. ¿Cuáles fueron los trayectos semánticos que marcaron el devenir de estas expresiones y sus posibles sentidos?

Desde un punto de vista etimológico es factible constatar la existencia de dos series latinas paralelas que derivarían de la misma raíz indo-europea. A través del término griego, $\alpha v \chi \omega$, la angustia y la ansiedad aludirían a cerrar, ahogar, obligar. La primera, "refiere a los verbos ango, angusto y angustio, el adjetivo angustus y una serie de sustantivos: angor, angustia (generalmente empleado en plural angustiae), angustita, angina y angustum. La segunda se inicia por el verbo anxio, contiene los adjetivos anxius, anxiosus, anxifer y los sustantivos anxia, anxietas et anxietudo" (20, p. 5). Bajo esta óptica, resulta casi imposible definir el sentido preciso de uno u otro término, porque ellos pueden ligarse tanto a un fenómeno físico, como la opresión, o a un fenómeno moral, como el tormento.

La oscilación entre lo físico y lo psíquico, así como las dificultades en la demarcación de la angustia respecto del miedo, atravesarán las tres lenguas que tuvieron un rol hegemónico en las definiciones psicopatológicas relativas al problema de la angustia: francés, alemán e inglés. Desde un punto de vista histórico el dominio semántico de la palabra angustia muestra, en estas tres lenguas, una variabilidad en lo que concierne a sus empleos contemporáneos.

Al interrogar la vía etimológica del término en francés, se constata que angoisse proviene de angustia. Bajo la forma de "angustus «estrecho», propr. « estrechamiento», de donde «desfiladero, pasaje estrecho», sentido conservado en fr. hasta el siglo XVI; «molestia de la respiración»" (21, p. 26). Asimismo, encontramos una referencia al orden moral, "del latín clásico « embarazo, dificultad » (...) Desde el siglo XVI It. (Latin) Angoscia «angustia, aflicción», esp. Congoja, del 
mismo sentido" (21, p. 26). En esta misma dirección, "angere « estrechar, oprimir, cerrar »" (22, p. 374). El término anxieté, por su parte, figura en "1190, retomado en 1564 en méd; generalizado en el siglo XVII; lat. anxietas « disposición natural a la inquietud » de anxius" (22, p. 433). Se consigna que el término anxieté posee el mismo radical que angoisse, sin embargo, y en un intento de diferenciación, se sostendrá que "la ansiedad es menos viva y más constante; es un estado de angustia, pero un estado disminuido" (22, p. 433).

En el siglo XVII se registra una expresión muy singular. Se trata de poire d'angoisse, "instrumento en forma de pera que los ladrones utilizaban como mordaza introduciéndola en la boca de sus víctimas, variedad de pera muy áspera" (23, p. 26). En inglés, este instrumento de tortura es designado como pear of anguish o choke pear (24), mientras que en alemán se denomina, descriptivamente, como Mundbirne, Folterbirne o Spreizbirne.

En la lengua alemana, si bien existen las palabras Angst y Furcht, el término Angst, está dotado de una particular polisemia e indeterminación, ya que, en un sentido general, refiere tanto a la angustia, como al miedo. Angst es un palabra compuesta que se desarrolla a partir del siglo VIII a través del alemán standard o alto alemán (Hochdeutsch), pasando por el antiguo alto alemán (Althohdeutsch) angust (25). Procede del indogermano angh (estrecho, angosto), también anghu (constrictivo, apremiante), y el sufijo st (perteneciente a). Sus raíces se remontan al latín, angustia, estrechura, apretura, término que, a su vez, remite al griego, angor, estrangulamiento, asfixia. Por lo tanto, Angst alude a la estrechez y a la constricción. Se emplea como sustantivo o adjetivo, aunque también se constata su empleo como verbo, ängstigen.

En lo que refiere a la lengua inglesa, la vía etimológica consigna las mismas raíces latinas y griegas. Sin embargo, podemos señalar que a pesar de la existencia de los términos anguish y anxiety, el empleo del vocablo anguish no será significativo en el dominio médico, siendo más bien una expresión de uso literario. En este último terreno, las aplicaciones resultarán diversas, enfatizando algunos de los matices incluidos en el campo semántico.

A diferencia de lo que ocurre en el terreno literario, el término anxiety se impondrá en la nomenclatura médica, alcanzando un estatuto conceptual y técnico. Esta opción se irradiará a las disciplinas psiquiátricas y psicológicas de manera masiva. No obstante, cabría precisar que, mientras que en la psiquiatría anglosajona la dicotomía anguish-anxiety tiene solamente una relevancia clínica secundaria (26), los términos angoisse, angst y angustia en la psiquiatría francesa, alemana y española, respectivamente, se asocian a un significado claramente delimitado, que apunta al estado paroxístico (4).

Este conflicto terminológico-conceptual entre angustia y ansiedad acompañará los desarrollos nosográficos más precoces de las problemáticas asociadas a 
ORIGINALES Y REVISIONES

la angustia, al intentar especificar las posibles relaciones entre el factor psíquico y el somático. Ya en 1902, Brissaud hacía un llamado a la comunidad médica y, en el desarrollo del debate, advertía: "No hay que confundir ansiedad y angustia. La ansiedad puede acompañar a la angustia, pero habiendo desaparecido esta última, la ansiedad persiste sola, como fenómeno puramente psíquico (...) la ansiedad, es, por definición, un estado del espíritu" (27, p. 44- 45).

Hacia mediados del siglo XIX, la reflexión de Kierkegaard enfrentará los dilemas asociados al campo semántico de la angustia y tendrá como tarea conceptualizar su singularidad, situando a la angustia como un concepto crucial en el abordaje de lo humano. Según Pierre, esta empresa resultaría inédita en la historia de la filosofía; si bien la angustia estaba presente en algunas reflexiones, siempre fue un tópico menor "con excepción de un dominio donde ella era vivida en el seno de una experiencia terrible, aquel de la mística- en el margen de la filosofía” (28, p. 3).

3. Kierkegaard y el concepto de angustia: algunos fragmentos sobre el problema del objeto y el tiempo.

Vigilius Haufniensis, el vigilante de Copenhague, intentará un análisis psicológico, con miras a interrogar el problema del pecado original, dando lugar a la elaboración de un concepto de angustia en el dominio filosófico.

Preguntándose por la condición previa al pecado original, Kierkegaard busca una explicación para el origen de la angustia, instalando una distinción fundamental. Afirmará de manera vehemente que "Casi nunca se ve tratado el concepto de la angustia dentro de la psicología; por eso mismo debo llamar la atención sobre la total diferencia que intercede entre este concepto y el del miedo, u otros similares. Todos estos conceptos se refieren a algo concreto, en tanto que la angustia es la realidad de la libertad en cuanto posibilidad frente a la posibilidad. Esta es la razón de que no se encuentre ninguna angustia en el animal, precisamente porque este, en su naturalidad, no está determinado como espíritu" (18, p. 50).

Desde el punto de vista del objeto, el miedo estaría referido a una cosa precisa, mientras que la angustia, por el contrario, no contaría con dicha referencia. ¿Es que la angustia no posee un objeto o es que Kierkegaard, distinguiendo la angustia del miedo, propone un nuevo objeto para la angustia? Algunos pasajes de su obra se inclinan por lo segundo, pudiendo entonces considerar su argumentación como una problematización del objeto de la angustia y el miedo. Lo que Kierkegaard llama "realidad de la libertad" o "posibilidad", ligarían a la angustia con lo indeterminado y lo anónimo. No obstante, una nueva precisión se vuelve necesaria, ya que "Si ahora, concretando más, preguntamos cuál es el objeto de la angustia, la 
respuesta no puede ser otra que la de siempre: ese objeto es la nada. Porque la angustia y la nada son siempre correspondientes entre si" (18, p. 116). De este modo, la nada llevaría a repensar el estatuto del objeto involucrado en la angustia.

Una particularidad psicológica de la angustia se sumará a la reflexión sobre el objeto, al destacar y subrayar su ambigüedad: "La angustia es una antipatía simpática y una simpatía antipática" $(18$, p. 117). Se resalta el carácter ambivalente y contradictorio de dicha experiencia, recalcando el movimiento de atracción y rechazo que participa de su dinamismo. En relación a ello, la figura del vértigo se vuelve ilustrativa: "A quien se pone a mirar con los ojos fijos en una profundidad abismal le entran vértigos. Pero ¿dónde está la causa de tales vértigos? La causa está tanto en sus ojos como en el abismo. ¡Si él no hubiera mirado hacia abajo! Así es la angustia el vértigo de la libertad; un vértigo que surge cuando, al querer el espíritu poner la síntesis, la libertad echa la vista hacia abajo por los derroteros de su propia posibilidad, agarrándose entonces a la finitud para sostenerse. En este vértigo la libertad cae desmayada (18, p. 73). Se incluyen entonces la contradicción, los opuestos, subvirtiendo las dimensiones de lo interno y lo externo.

Así, la angustia, como afirma Jolivet, "instalada en la soldadura de la posibilidad y de la realidad, revela el existente a sí mismo, le propone un yo a realizar" $(29$, p. 59). Bajo estas coordenadas, la angustia podría ser considerada como la más abrumadora de las categorías. Ese cuerpo que es exceso y absoluto, infinito y vacío, confronta a los sujetos con la autenticidad, por lo que podría considerársela como una vivencia que destruyendo todas las finitudes apela a la individualidad (30).

El Concepto de la angustia ofrecerá otros elementos de gran importancia, ya que el problema del objeto involucrará, necesariamente, una dimensión temporal.

Si el espacio se transformó en algo fundamental, en la medida que la ausencia o la presencia de un objeto actuaba como criterio diferencial, el tiempo será un registro a partir del cual establecer distinciones. Kierkegaard sostendrá que la angustia siempre estaría referida al futuro, en cuanto ella involucra lo posible. El pasado sólo producirá angustia en cuanto invoque lo futuro, ya que "Si me angustio por una desgracia pasada no es precisamente en cuanto pasada, sino en cuanto puede repetirse, es decir, hacerse futura. Si tengo angustia por una mala acción pasada, entonces es que no la he relacionado esencialmente conmigo en cuanto pasado, sino que hay algo en mi vida que de una manera más o menos subrepticia le impide ser pasada. Pues si realmente fuese pasada, entonces no podría angustiarme, sino sólo arrepentirme" $(18$, p. 109). La angustia, como un movimiento que se proyecta al avenir, diría relación con la expectativa, la espera, la posibilidad y la indeterminación.

En un intento por aprehender el carácter singular y excepcional de esta experiencia, se consigna que la angustia es una problemática que no se encuentra en los animales. La distinción entre angustia y miedo, las relaciones de la angustia 
ORIGINALES Y REVISIONES

con la libertad y la posibilidad, sustentan un concepto que remite a una experiencia exclusivamente humana, por lo que "El hombre no podría angustiarse si fuese una bestia o un ángel. Pero es una síntesis, y por eso puede angustiarse. Es más, tanto más perfecto será el hombre, cuanto mayor será la profundidad de su angustia. Sin embargo, esto no hay que entenderlo - como suele entender la mayoría de la gente - en el sentido de una angustia por algo exterior, por algo que está fuera del hombre, sino de tal manera que el hombre mismo sea la fuente de la angustia" (18, p. 181).

La angustia no podría ser reducida a una referencia corporal o instintiva, ya que su producción aparece ligada a la condición humana. Si bien su irrupción compromete a un cuerpo, no es posible definirla como un recurso adaptativo. "La angustia no es una categoría de la necesidad, pero tampoco lo es de la libertad. La angustia es una libertad trabada, donde la libertad no es libre en sí misma, sino que está trabada, aunque no trabada por la necesidad, más por si misma" (18, p. 59).

Tras estas tesis relativas a la angustia se impone la pregunta respecto de ¿Cuáles podrían ser los alcances y las consecuencias de la reflexión filosófica para el campo médico? La psiquiatría acogerá ciertos aspectos de la teorización propuesta por Kierkegaard, ofreciendo una lectura pragmática e instrumental de algunas de sus distinciones.

4. Las nuevas versiones de la angustia: innovaciones psicopatológicas de la segunda mitad del siglo XIX

Hacia mediados del siglo XIX la angustia aún no había alcanzado el rango oficial de problema médico psicopatológico. El retraso en su reconocimiento se verá graficado, como advierte Pichot (31), en la ausencia de una definición exclusivamente psicopatológica a lo largo de los cien volúmenes del Dictionnaire encyclopédique des sciences médicales (32), obra de referencias para la escena médica francesa de la época.

Pese a ello, en ciertos trabajos pioneros comenzarán a resonar los grandes ejes consignados por Kierkegaard, aunque la referencia no sea evidente. Por una parte, se identifica la participación de afectos, sentimientos y emociones en la producción de fenómenos psicopatológicos y, por otra, se comienzan a proponer distinciones en el universo angustioso, tomando como criterios a las dimensiones espacial y temporal, indicaciones que habían resultado útiles en la diferenciación del miedo.

En este sentido, Morel sostenía que "designando el factor de emociones generales de nuestro ser bajo el nombre de sentido emotivo no hemos creado una entidad psicológicamente abstracta (...) Hablamos de un sentido que existe real- 
mente, y del cual numerosas manifestaciones surgen en el estudio de fenómenos del pensamiento y en aquel de la producción de nuestros actos" (33, p. 126). La nueva categoría de délire émotif (delirio emotivo) referiría a "hechos de impresionabilidad y de emotividad" (5, p. 385) que estarían a la base del cuadro. Aunque su nueva entidad nosológica referiría a lo delirante, advertirá que si bien toda locura es un delirio, no todo delirio es una locura (33). Los términos angustia o ansiedad, no serán consignados de manera particular, no obstante, el valor de los afectos, sentimientos y emociones serán invocados en la comprensión de fenómenos psicopatológicos, mediante la categoría de lo emotivo.

Tras el primer gesto emprendido por Morel, la angustia y el miedo serán abordados de manera directa por Westphal y Legrand Du Saulle, quienes propondrán nuevas categorías para abordar fenómenos clínicos relativos al orden fóbico.

Mediante el estudio de algunos casos clínicos Westphal reportaba un fenómeno novedoso en el cual "el enfermo es súbitamente afectado por vértigos y angustias, desde que debe atravesar un espacio vacío" (34, p. 175). Esto permitiría sostener que "ese terror de atravesar plazas y calles representaba en ese punto el fenómeno principal" (6, p. 26), por lo que sería justificado proponer el término agoraphobie (agorafobia), ya que el hecho central es el miedo (phobos) de las plazas (agora).

Legrand du Saulle, criticando la proposición de Westphal, se declarará en desacuerdo con la categoría promovida por los alemanes, debido a su referencia exclusiva a las plazas, ya que "las observaciones clínicas de los autores y las mías propias - dirá - establecen, por el contrario (...) que los enfermos tienen miedo del espacio, del vacío" $(35$, p. 6). Como alternativa propondrá la categoría de peur des espaces (miedo a los espacios) "un estado neuropático muy particular, caracterizado por una angustia, una impresión ansiosa viva, o incluso un verdadero terror, produciéndose súbitamente en presencia de un espacio dado. Este accidente es esencialmente emotivo. No se acompaña jamás de pérdida completa del conocimiento o de caída, y es totalmente distinto de la hipocondría, del vértigo epiléptico, del vértigo estomacal, del vértigo gotoso y, sobretodo, de la neuropatía cerebro- cardíaca" $(35$, p. 5). Dicha enfermedad es compatible con el más pleno estado de salud. Es en el enfrentamiento de un espacio determinado donde surge "una angustia súbita, un cerramiento instantáneo del corazón. El enfermo, presa de una indefinible emoción, se encuentra aislado del mundo entero ante la apariencia del vacío que se le ofrece y se espanta sin medida, pese al poco fundamento de su temor y a pesar de las más sabias y las más tranquilizadoras exhortaciones que se dirige espontáneamente a sí mismo; se siente como aniquilado" (35, p. 7).

Los aportes de Westphal y Legrand du Saulle dejan en evidencia de qué modo las discusiones sobre la angustia y el miedo se instalaron en la escena médica para referirse a un estado mental. Se podía tratar de una experiencia humana compren- 
ORIGINALES Y REVISIONES

sible, pero también podía dar lugar a un cuadro patológico o, incluso, conducir a la insania (4).

A comienzos del siglo XX las distinciones sobre la angustia aportadas por Kierkegaard, tomarán una clara definición y se inscribirán de una manera sistemática y operativa en los escritos psicopatológicos.

En 1913, Karl Jaspers emprendió el ambicioso proyecto de renovar la psicopatología de su época (36). Preguntándose por las posibles relaciones entre la filosofía y la psicopatología sostendría que "en toda ciencia viva la filosofía es eficaz, que la ciencia sin filosofía no es fecunda, no es verídica, sino que sólo puede ser exacta" (36, p. 848). Bajo estas consideraciones advierte que el no hacer conscientes los supuestos filosóficos, hace que estos se plasmen inadvertidamente en el pensamiento y el lenguaje científico, volviendo toda reflexión oscura. De este modo, "la filosofía crea el espacio en el que tiene lugar todo saber, adquiere medida y límites y el terreno, en que puede mantenerse y volverse práctico, recibe así contenido y significación" (36, p, 848). Tras el elogio de poetas y artistas, y habiendo declarado como referencias ineludibles la filosofía de Platón, Aristóteles y los estoicos; el aporte de San Agustín, Montaigne, La Bruyère, La Rochefoucauld, Vauvernages, Chamfort y Pascal; subrayando el aporte de Hegel, definirá, finalmente, a Kierkegaard y Nietzsche como "los más grandes de todos los psicólogos comprensivos" (36, p, 848).

Denunciando la poca precisión con que la psicopatología y la psicología han tratado el problema de los sentimientos, en comparación con otros tópicos tales como los de consciencia, pensamiento o volición, se propone establecer principios organizadores y diferenciadores que permitan superar la tendencia a elaborar listados o colecciones de sentimientos.

Entre las categorías definidas para tales fines postulará la categoría de Sentimientos sin objeto, sentimientos que "tienen primero que buscar o producir su objeto para llegar a la autocomprensión” (36, p. 130). Bajo esta referencia se propone distinguir, en primer lugar, la particularidad de la angustia, un sentimiento que califica de frecuente y torturante, afirmando que "el miedo es dirigido a algo, la angustia es atópica" (36, p, 130). La angustia refiere a una sensación sentimental que compromete al corazón y tiene un carácter vital, pudiendo dividirse en "angustia estenocárdica (en la angina pectoris) y en angustia de sofocación (en el hambre de aire, por ejemplo, en los trastornos descompensados de la circulación)" (36, p. 130- 131). Pero será catalogada como un "estado psíquico primario, en analogía con la angustia vital siempre referida a la existencia en su conjunto, penetrándola y dominándola" (36, p. 131). Su emergencia involucraría al cuerpo, imprimiendo sensaciones relativas a la presión, la sofocación y la estrechez, y, a su vez, promoviendo una inquietud que es vivida como un sentimiento interno de excitación. Llegará incluso a proponer una angustia existencial, "una disposición básica del 
existir que se vuelve notoria en situaciones fronterizas, ese origen de la existencia, no es captable fenomenológicamente" (36, p. 131).

De este modo, anudando la reflexión filosófica y el material psicopatológico, Jaspers logra situar una distinción fundamental, al separar la angustia del miedo. Destacando aun más la relevancia de tal diferenciación, Pichot subrayará "que en danés Angest (moderno Angst) es tomado del alemán y que, en las dos lenguas, significa al mismo tiempo angustia y miedo. Esto vuelve aún más destacable que Kierkegaard, como más tarde Jaspers, hayan propuesto un criterio psicopatológico separando radicalmente los dos conceptos $(20$, p. 7$)$.

Podría pensarse que esta será una distinción que sólo alcanzará relevancia en el ámbito psiquiátrico. Sin embargo, será el propio Freud quien la hará suya, al afirmar que el término " "angustia» se refiere al estado y prescinde del objeto, mientras que «miedo» dirige la atención justamente al objeto" (37, p. 360). En 1926 agregará, "La angustia tiene un inequívoco vínculo con la expectativa (...) Lleva adherido un carácter de indeterminación y ausencia de objeto; y hasta el uso lingüístico correcto le cambia el nombre cuando ha hallado un objeto, sustituyéndolo por el de miedo \{Furcht\}" (38, p. 154).

Así, podemos apreciar como la reflexión filosófica de Kierkegaard no sólo aportó distinciones útiles en torno a la diferenciación entre angustia y miedo en el ámbito filosófico, sino que dichas puntualizaciones se constituyeron como elementos de referencia para construir e inscribir, en el marco clasificatorio, una serie de fenómenos que atravesarán las principales categorías diagnósticas de la psiquiatría de la segunda mitad del siglo XIX.

\section{Discusión}

Pese a la significativa presencia de la angustia en el ámbito de lo humano, su incorporación al campo psicopatológico se producirá de manera tardía, en comparación con otras categorías de relevancia.

Históricamente el registro semántico ofrece figuras tales como el dolor, lo moral y, en un sentido más amplio, el sufrimiento. Atravesará los siglos evocando la inquietud, la humillación y la desesperación, alcanzando incluso, el orden de la culpabilidad. Está diversidad de acepciones y usos encontrará su correlato en el ámbito médico, donde la angustia será un fenómeno que recorrerá el campo patológico en su conjunto. Se trata de un fenómeno que inicialmente será asociado a distintos órganos como un signo de malestar, para luego alcanzar una definición psicopatológica.

Más allá del marco teológico, la reflexión llevada a cabo por Kierkegaard en su obra El concepto de la angustia introducirá una serie de distinciones que vere- 
ORIGINALES Y REVISIONES

mos reflejadas en el campo nosográfico de la psiquiatría. Entre estas innovaciones podemos subrayar el llamado a situar a la angustia como una experiencia inherente a la condición humana. Asimismo, se advertirá sobre la necesidad de diferenciar a la angustia del miedo y de sus conceptos afines (pánico, horror, temor, etc.). Las referencias al objeto y la temporalidad serán los aspectos que la psicopatología de fines del siglo XIX y comienzos del XX establecerá como variables rectoras en la diferenciación de la angustia y el miedo. El miedo, desde esta perspectiva, será un sentimiento referido a la presencia de un objeto, mientras que la irrupción de la angustia se ligará a la ausencia de este. Dichas consideraciones se plasmarán en algunas de las categorías precursoras del campo de la angustia, tales como las de delirio emotivo, agorafobia, miedo a los espacios, para luego ser abordadas de manera directa y específica en la obra de Karl Jaspers.

La psiquiatría contemporánea incorporará estas distinciones, enlazándolas con procesos perceptivos, ideativos y, por cierto, con todo el correlato neurofisiológico involucrado en el fenómeno de la angustia. Si bien esta aplicación será útil en la lógica clasificatoria, las nuevas versiones de la angustia privilegiarán el correlato neurovegetativo, relegando a un segundo plano los aspectos subjetivos, los cuales se consignarán como ideas relativas a la muerte, la locura o la enfermedad o serán identificados en fenómenos tales como la desrealización o la desperzonalización.

El carácter excepcional de la angustia se verá redefinido por la psicopatología bajo los parámetros de la intensidad, la frecuencia y la duración, enfatizando el carácter descriptivo del fenómeno. Se enfatizará su carácter sintomático con miras a establecer un posible trastorno. Expresiones tales como crisis de angustia, trastorno de angustia - comúnmente designados como crisis de pánico o trastorno de pánico- mostrarán un carácter eminentemente pragmático despojando al fenómeno angustioso de su referencia existencial. Si a esto sumamos el privilegio por el término ansiedad, lo que se verá reforzado será el correlato neurofisiológico y el aspecto conductual involucrado en el fenómeno.

Bajo estas consideraciones las reflexiones filosóficas quedarán relegadas a un hecho de orden histórico, tras los nuevos referentes clasificatorios. 
ORIGINALES Y REVISIONES

\section{BIBLIOGRAFIA:}

(1) Pigeaud, J. La maladie de l'âme. Étude sur la relation de l'âme et du corps dans la tradition médico-philosophique antique. Paris: Les Belles Lettres, 2006.

(2) López-Ibor Aliño, J. J. \& Valdés Miyar, M. (dir.). DSM-IV-TR. Manual diagnóstico y estadístico de los trastornos mentales. Barcelona: Masson, 2002.

(3) Organización Panamericana de la Salud. CIE- 10. Clasificación estadística internacional de enfermedades y problemas relacionados con la salud: décima revisión, 2003.

(4) Berrios, G. Historia de los síntomas de los trastornos mentales. La psicopatología descriptiva desde el siglo XIX. México: Fondo De Cultura Económica, 2008.

(5) Morel, Benedickt. Du délire émotif. Névrose du système nerveux ganglionnaire viscéral. Archives générales de médecine. Paris : Mémoires originaux, 1886.

(6) Westphal, C. «"Die agoraphobie", eine neuropatische Erscheinung» (1872), Gesammelte Abhandlungen, Band 1, Berlin: Psychiatrische Abhandlungen, 1892. En: Vallon, S. L'espace et la phobie. La peur de la peur- 1. Paris : Éres, 1996.

(7) Krishaber, M. « Cérebro- cardiaque (névropathie) », en A. Dechambre y L. Lereboullet (eds.) Dictionnaire Encyclopédique de Sciences Médicales, Vol. 14. Paris : Masson, 1873 ; 100- 142.

(8) Legrand Du Saulle, H. Etude cliniques sur la peur des espaces (Agoraphobie des Allemands). Névrose Emotive. Paris: Delahaye et Cie. 1878.

(9) Da Costa, J. M. On irritable heart: a clinical study of a form of functional cardiac disorder and its consequences. Am. J. Med. Sci. 1871; (121): 17- 52.

(10) Beard, G. M. A practical treatise on nervous exhaustion (neurasthenia), its symptoms, nature, sequences, treatment. New York: Wood, 1880.

(11) Beard, G. M. American nervousness, its causes and consequences, a supplement to "Nervous exhaustion (neurasthenia)". New York: G. P. Putnam's sons, 1881.

(12) Levillain, F. La neurasthénie. Maladie de Beard. Paris: A. Maloine, libraire-éditeur, 1891.

(13) Kaan, H. Der neurasthenische Angsteffekt bei Zwangsvorstellungen und der primordiale Grübelzwang. Leipzig: Deutike, 1892.

(14) Freud, S. "Sobre la justificación de separar la neurastenia de un determinado sindrome en calidad de «neurosis de angustia»" (1895), Vol. 3, p. 85- 115. Obras Completas. Buenos Aires: Amorrortu, 1996.

(15) Freud, S. "A propósito de las críticas a la «neurosis de angustia»" (1895), Vol. 3, p.118- 138. Obras Completas. Buenos Aires: Amorrortu, 1996.

(16) Hecker, E. Zur Behandlung der neurasthenischen Angstzustände. Berliner Klin. Wochenschr, 1892; (29): 1195- 1197.

(17) Hecker, E. Über larvative und abortive Angstzustände bei Neurasthenie. Central- blatt F. Nervenheilk. Psychiatr 1893; (16): 565- 572.

(18) Kierkegaard, S. El Concepto de la angustia. Buenos Aires: Ediciones Libertador, 2004.

(19) López- Ibor, J. J. La angustia vital. Patología General Psicosomática. Madrid: Paz Montalvo, 1950.

(20) Pichot, P. D'où Vient L'attaque De Panique? En: Lemperière, Th.(dir.) Le Trouble Panique. Paris: Acanthe, Masson, Smithkline Beecham, 1998.

(21) Dictionnaire étymologique de la langue française. Bloch, O.; Von Wartburg, W. Paris: P. U. 


\section{ORIGINALES Y REVISIONES}

F., 2004.

(22) Dictionnaire Le Grand Robert de la langue française. Paris: Dictionnaires Le Robert, 1991.

(23) Le Dictionnaire du français classique. Le XVIIe Siècle. Dubois, J ; Lagane, R ; Lerond, A. Paris: Larousse, 1992.

(24) Calvi, F.; Baragnes, R. \& Lacassagne, A. Histoire Générale des Larrons. Divisée en 3 Livres. Paris: Rolin Baragnes, 1631.

(25) Kluge, F. Etymologisches Wörterbuch Der Deutschen Sprache. Berlin: de Gruytre, 1999.

(26) Lewis, A. Problems presented by the ambiguous word «anxiety» as used in psychopathology, The Israel Annals of Psychiatry und Related Disciplines, 1967; (5): 105-121.

(27) Congrès des médecins aliénistes et neurologistes de France et des pays de langue française. XII session tenue à Grenoble du $1^{\circ}$ au 7 août 1902. Vol 2. Paris: G. Masson, Editeur Libraire de l'académie de médicine, 1902.

(28) Pierre, M. L'émergence du concept d'angoisse a l'époque du romantisme Allemand. Paris: Presses Universitaires Du Septentrion, 2002.

(29) Jolivet, R. Las doctrinas existencialistas. Madrid: Editorial Gredos, 1976.

(30) Wahl, J. Kierkegaard. L'un devant l'autre. Paris : Hachette Littératures, 1998.

(31) Pichot, P. Kierkegaard et la naissance du concept de l'angoisse. Confrontations Psychiatriques, 1995; (36): 27- 33.

(32) Dictionnaire encyclopédique des sciences médicales. T. 5, ANG- ARB. Paris: G. Masson, P. Asselin, 1866.

(33) Morel, B. Traité théorique et pratique des maladies mentales considérés dans leur nature, leur traitement et dans leur rapport avec la médicine légale de aliénés. T. 2. Paris: Vitor Masson Libraire, 1853.

(34) Westphal, C. Annales Médico- Psychologiques, 5e série. T. 10: Paris, 1873.

(35) Legrand du Saulle, H. Etude cliniques sur la peur des espaces (agoraphobie des Allemands). Névrose émotive. Paris: Delahaye et Cie, 1878.

(36) Jaspers, K. Psicopatología General. $2^{a}$ ed. Mexico: Fondo de Cultura Económica, 1993.

(37) Freud, S. Conferencias de introducción al psicoanálisis (1916- 1917). 25ª Conferencia. La angustia. Obras Completas, Vol. XVI. p. 357- 374. Buenos Aires: Amorrortu, 1998.

(38) Freud, S. Inhibición, síntoma y angustia. Obras Completas. Vol. XX. p. 71- 163. Buenos Aires: Amorrortu, 1998. 